\title{
Peanut-Like Hematite Prepared by a New Facile Hydrothermal Process for Removal of $\mathrm{As}(\mathrm{V})$
}

\author{
Chengjin Zhang ${ }^{1} \cdot$ Shaoyi Jia ${ }^{1}$ Songhai $\mathrm{Wu}^{1} \cdot$ Yong Liu $^{2}$
}

Received: 9 February 2018 / Revised: 22 March 2018 / Accepted: 4 April 2018 / Published online: 17 May 2018

(c) The Author(s) 2018

\begin{abstract}
Peanut-like hematite has been prepared by a new facile hydrothermal method and applied in the adsorption removal of As(V). The structural features of the as-prepared hematite were characterized systematically by X-ray diffraction, X-ray photoelectron spectroscopy, Brunauer-Emmett-Teller, scanning electron microscopy, energy-dispersive X-ray spectroscopy mapping, Fourier transform infrared spectroscopy, and transmission electron microscopy. Results showed that the morphologies of hematite could be tuned to spindle-like, oval-like, and cantaloupe-like shapes by adjusting the hydrothermal conditions. The peanut-like hematite formation followed a five-step route. At $\mathrm{pH}=3$, the adsorption amount of $\mathrm{As}(\mathrm{V})$ over peanut-like hematite reached $13.84 \mathrm{mg} / \mathrm{g}$, and the adsorption kinetic process corresponded to the pseudo-second-order kinetic model. The peanut-like hematite also showed partial selectivity over As(V) in the hydrosphere. This method can be a reference for the preparation of other architectural metal oxide materials.
\end{abstract}

Keywords Peanut-like hematite $\cdot$ Arsenic adsorbent $\cdot$ Nanoparticle

\section{Introduction}

Arsenic contamination has led to serious environmental problems because of its severe toxicity and risk to human health [1]. Thus, removing arsenic from aqueous environment is of critical importance. Recently, significant efforts have been devoted to removing arsenic pollutants from water systems by various strategies, including coagulation, adsorption, and reverse osmosis [2]. Among these strategies, adsorption is the simplest and most effective method for arsenic removal. Adsorption efficiency is highly dependent on adsorbent characteristics. Hence, high-efficient adsorbents must be urgently developed.

Hematite is an attractive and environment friendly adsorbent owing to its chemical stability, easy fabrication, and unique optical and electric properties; it is widely applied in adsorption, chemical catalysts, lithium-ion batteries, gas sensors, and electrode materials [3]. Given the specific

Songhai $\mathrm{Wu}$

wusonghaitjuedu@163.com

1 School of Chemical Engineering and Technology, Tianjin University, Tianjin 300350, China

2 School of Chemistry and Chemical Engineering, Tianjin University of Technology, Tianjin 300384, China interactions between hematite and oxyanions of arsenic species, hematite can be used as an adsorbent to remove arsenic [4]. Adsorption efficiency is related to the specific surface area, morphology, and surface groups of adsorbents [5]. Different hematite morphologies, such as dots [6], rods [7], wires [8], arrays [9], tubes [10], belts [11], disks [12], rings [13], and flower-like shapes [14], have been obtained. Sugimoto et al. [15] have synthesized hematite particles by the sol-gel method. Jia et al. [16] have synthesized hematite with a controllable size. However, the synthesis processes are tedious, and the adsorptive efficiency of arsenic remains unsatisfactory $[17,18]$. Thus, a facile method must be developed to synthesize diverse hematite nanostructures.

In this study, we report for the first time a new facile hydrothermal process using 5-sulfoisophthalate acid sodium salt (5-SSIPA) to synthesize peanut-like hematite and remove $\mathrm{As}(\mathrm{V})$. The possible formation process of such peanut-like hematite was put forward, and the efficiency and selectivity for the $\mathrm{As}(\mathrm{V})$ removal of the peanut-like hematite were also studied. 


\section{Experimental}

All procured chemicals were of analytical grade and used without further purification. Typically, $0.02 \mathrm{~mol}$ $\mathrm{FeCl}_{3} \cdot 6 \mathrm{H}_{2} \mathrm{O}$ and $0.02 \mathrm{~mol} 5$-SSIPA were dissolved in $60 \mathrm{~mL}$ deionized water in a $100 \mathrm{~mL}$ Teflon-lined stainless steel autoclave and heated at $190{ }^{\circ} \mathrm{C}$ for $6 \mathrm{~h}$. Afterward, the autoclave was cooled to room temperature naturally, and a reddish brown powder was obtained by centrifugation and washed at least thrice with deionized water. Then, the reddish brown powder was dried by a freeze dryer and stored in a glass vial.

The morphology and distribution of oxygen and iron elements were demonstrated by scanning electron microscopy (SEM). The valence states of Fe were analyzed by X-ray photoelectron spectroscopy (XPS). The crystal morphology was demonstrated by transmission electron microscopy. The phase reflection was analyzed by X-ray diffraction (XRD) with $\mathrm{CuK}_{\alpha}(\lambda=0.15406 \mathrm{~nm})$ radiation. The infrared optical properties were demonstrated by Fourier transform infrared spectroscopy (FT-IR). The Brunauer-Emmett-Teller (BET) surface area and pore size distribution were analyzed by $\mathrm{N}_{2}$ adsorption-desorption isotherm.

The peanut-like hematite with a concentration of $1 \mathrm{~g} / \mathrm{L}$ was equilibrated in $30 \mathrm{~mL} \mathrm{NaCl}$ solution with concentration of $0.1 \mathrm{~mol} / \mathrm{L}$ for $2 \mathrm{~h}$. Then, $\mathrm{As}(\mathrm{V})$ and $\mathrm{HCl}(0.1 \mathrm{~mol} / \mathrm{L})$ were added into the solution with an initial concentration and $\mathrm{pH}$ of $5100 \mathrm{mg} / \mathrm{L}$ and 3.0, respectively. The solutions were placed in a water bath shaker at $160 \mathrm{r} / \mathrm{min}$ and $25^{\circ} \mathrm{C}$. A total of $1 \mathrm{~mL}$ filtrate was obtained from the initial solutions at concentrations of 5 and $100 \mathrm{mg} / \mathrm{L}$ after a regular time $(5,10,15,25$, and $35 \mathrm{~min})$ and $12 \mathrm{~h}$, respectively. As $(\mathrm{V})$ concentrations were tested by atomic fluorescence spectrometry (Rayleigh, China) as referred to the work of Liu et al. [19].

\section{Results and Discussion}

Figure 1 shows the XRD, XPS of $\mathrm{Fe} 2 \mathrm{p}$, and $\mathrm{N}_{2}$ adsorption-desorption isotherm analysis of the samples. All peaks in Fig. 1a can be well matched to hematite (JCPDS No. 33-0664), and no characteristic peaks of other iron oxides were detected. The sharp peaks demonstrate the highly crystallized structure of the as-prepared hematite. The spectrum of $\mathrm{Fe} 2 \mathrm{p}$ in Fig. 1b demonstrates the two notable peaks at ca.710.9 and ca.723.7 eV, which correspond to $\mathrm{Fe} 2 \mathrm{p}_{3 / 2}$ and $\mathrm{Fe} 2 \mathrm{p}_{1 / 2}$ of $\mathrm{Fe}^{3+}$ in hematite, respectively [20]. Figure 1c illustrates the $\mathrm{N}_{2}$ adsorption-desorption isotherms and pore size distribution. The results demonstrate that the loop of
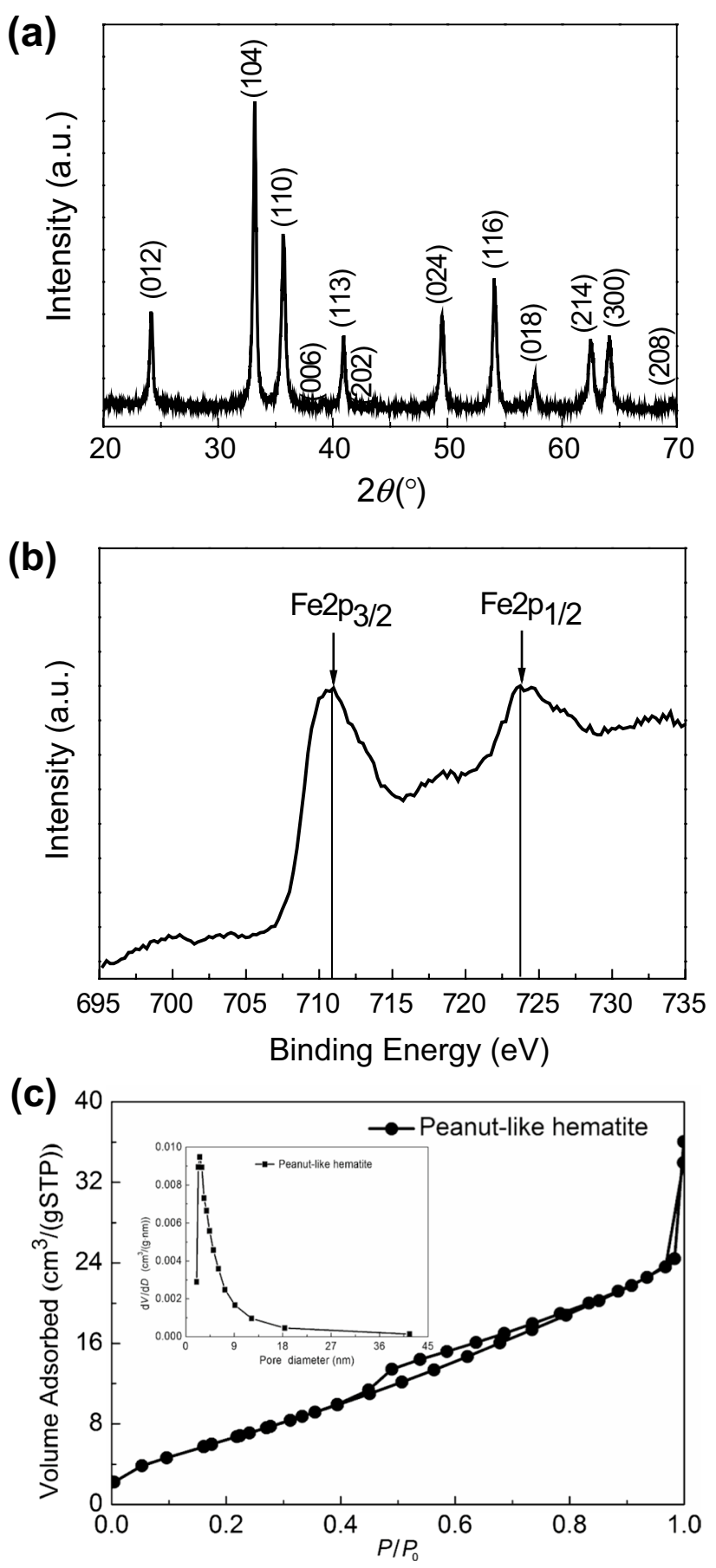

Fig. 1 a XRD, b XPS of Fe2p, and $\mathbf{c} \mathrm{N}_{2}$ adsorption-desorption isotherms (inset picture is pore size distribution) of the as-obtained products

the sample agrees with the type IV isotherm, suggesting the presence of mesopores in the sample [21]. The BET surface area, pore volume, and pore size of the sample were measured as $26.7519 \mathrm{~m}^{2} / \mathrm{g}, 0.05 \mathrm{~cm}^{3} / \mathrm{g}$, and $3.93 \mathrm{~nm}$, respectively. The results showed that the samples comprised hematite of a large surface area. 
SEM, high-resolution TEM (HRTEM), and energy-dispersive X-ray spectroscopy (EDX) mapping of iron and oxygen elements were obtained to further demonstrate the morphology, lattice information, and element distribution of the hematite samples, respectively. Figure 2 a demonstrates the high-yield growth and ordered distribution of peanut-like hematite. Figure $2 \mathrm{~b}$ shows the highly symmetric morphology of hemispherical ends with a thin waist of a single peanut structure. The length and width in the middle and ends of the peanut-like hematite reached ca. 3.79, 2.08 , and $2.59 \mu \mathrm{m}$, respectively. The surface sample consisted of irregular granules. Figure $2 \mathrm{c}$ shows the HRTEM image, demonstrating the single-crystal feature and high crystallinity of the sample. The results were consistent with the XRD analysis, and the featured $0.27 \mathrm{~nm} d$-spacing corresponded to that of (104). Figure $2 \mathrm{~d}$ and e shows the even distribution of oxygen and iron, respectively, on the surface of the single structural peanut-like sample. The results demonstrated that the hematite sample possessed unique symmetric peanut-like morphology with fine crystalline.

The morphologies of the final samples were affected by the components of the reaction solutions. In processing the zero-5-SSIPA component, spindle-like hematite with irregular particles in the center and sharp rod bundles in the ends was observed, as shown in Fig. 3a. The morphology changed significantly when $0.005 \mathrm{~mol}$ 5-SSIPA was added into the system; Fig. $3 b$ shows that cantaloupe-like hematite was obtained. Figure $3 \mathrm{c}$ and $\mathrm{d}$ displays the influence of $\mathrm{Fe}^{3+}$ contents $(0.03$ and $0.05 \mathrm{~mol}$, respectively). With increasing $\mathrm{Fe}^{3+}$ contents, the sample size enlarged and became inhomogeneous. The reason may be the higher concentration, which leads to easy aggregation and formation of over-peanut-like morphology. Thus,
Fig. 2 a, b SEM, c HRTEM, and EDX mapping of $\mathbf{d} \mathrm{O}$ and $\mathbf{e}$ Fe of peanut-like hematite
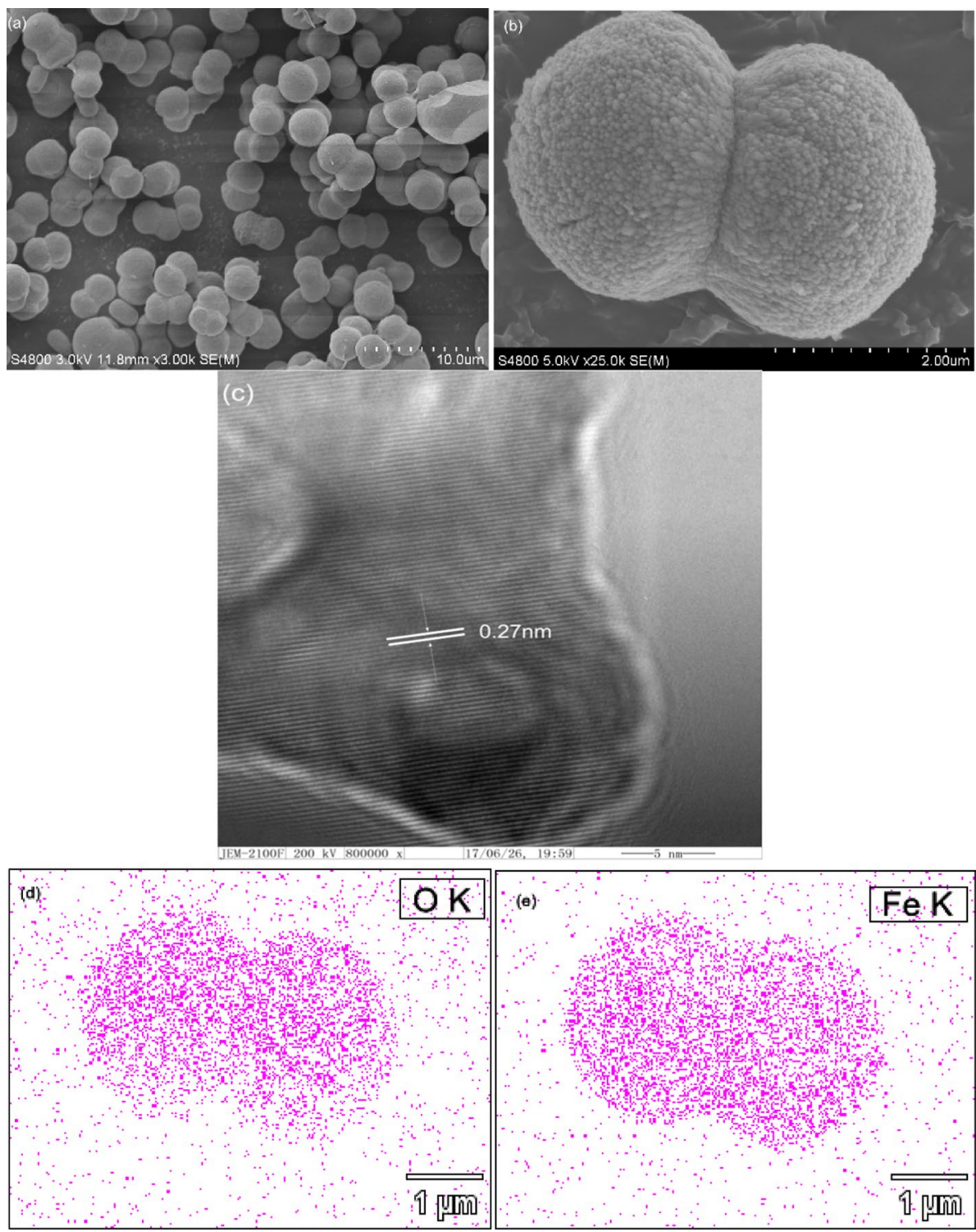
Fig. 3 SEM images of samples with different components (a, zero 5-SSIPA, b $0.005 \mathrm{~mol}$ 5-SSIPA, c $0.03 \mathrm{~mol} \mathrm{Fe}^{3+}$, and d $0.05 \mathrm{~mol} \mathrm{Fe}^{3+}$ )
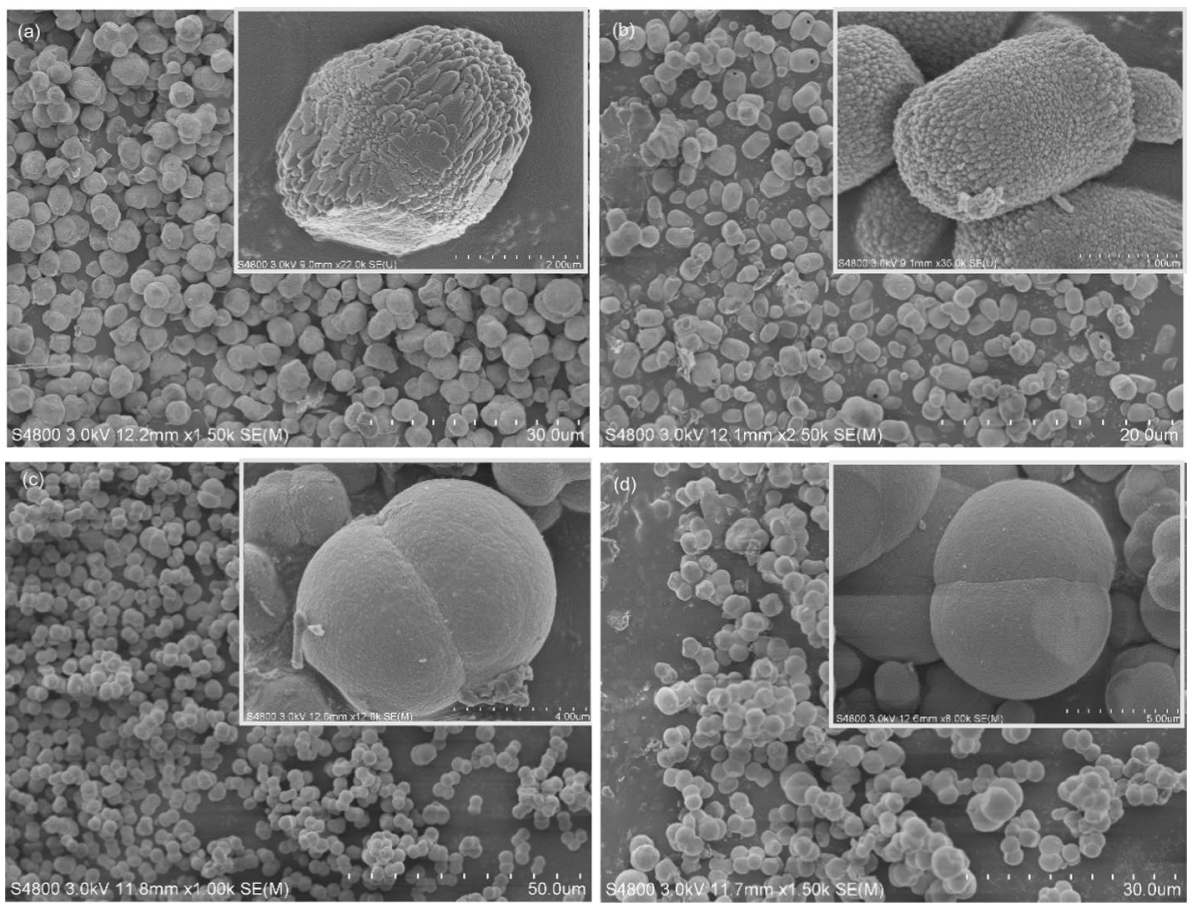
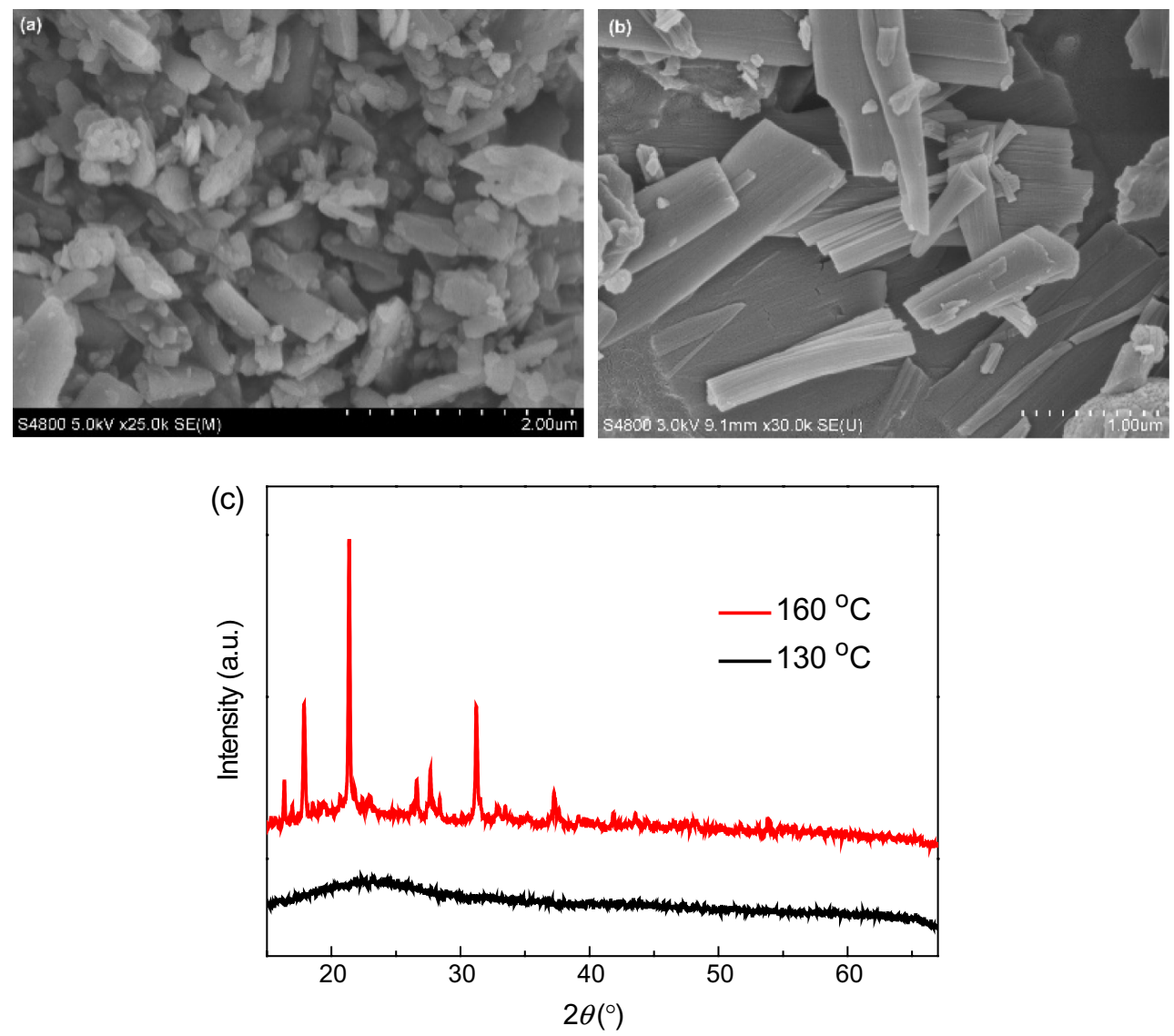

Fig. 4 SEM images (a $130^{\circ} \mathrm{C}$ and $\mathbf{b} 160^{\circ} \mathrm{C}$ ) and $\mathbf{c}$ XRD patterns of the samples at different hydrothermal temperatures 

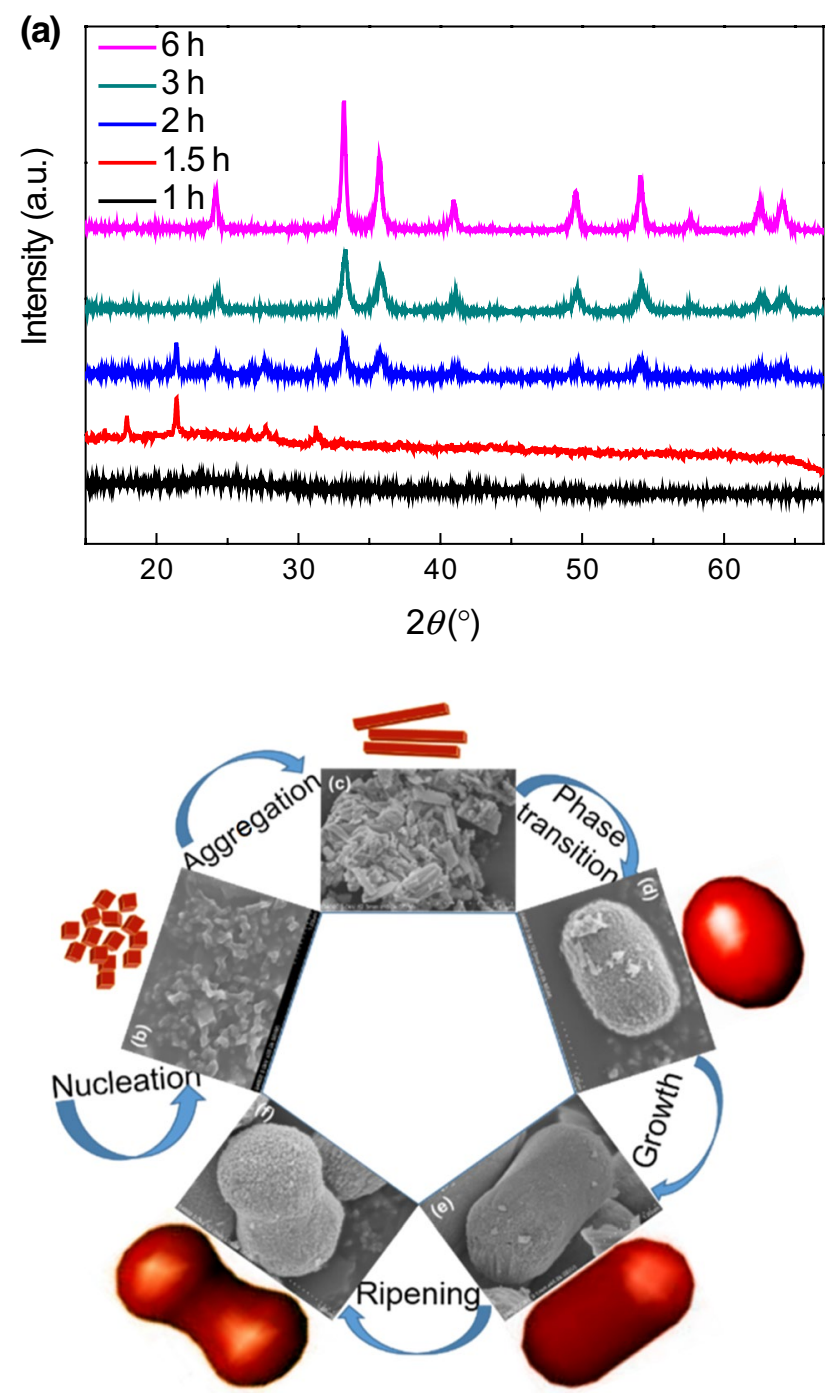

Fig. 5 a XRD patterns and SEM images of samples for different hydrothermal times (b 1, c 1.5, $\mathbf{d} 2$, e 3, and $\mathbf{f} 6 \mathrm{~h}$ ) and the formation process of peanut-like hematite

the morphologies of the final hematite samples could be adjusted by the contents of components in the solution system.

In addition to the solution component, hydrothermal temperature was also vital to the morphology of the final samples. Rod-like samples are obtained at $160{ }^{\circ} \mathrm{C}$ in Fig. 4b. The XRD patterns in Fig. 4c show that the samples comprised iron hydroxide $\left(\mathrm{Fe}(\mathrm{OH})_{3}\right.$, JCPDS No. 22-0346), goethite $(\alpha-\mathrm{FeO}(\mathrm{OH})$, JCPDS No. 29-0713), iron oxide hydroxide (FeOOH, JCPDS No. 18-0639), and a part of hematite. The as-obtained samples showed no notable morphology at $130{ }^{\circ} \mathrm{C}$, and the XRD pattern corresponded to that in Fig. $5 \mathrm{~b}$ for a hydrothermal period of $1 \mathrm{~h}$. The two samples were not hematite according to the analysis of XRD patterns. Thus, hydrothermal temperature can highly affect the final product and morphologies by affecting nucleation and aggregation to some extent, and a lower temperature is disadvantageous for crystallization.

Combining the XRD patterns in Fig. 5a and SEM images in Fig. 5b-f, we proposed and simulated the process of a five-step route consisting of nucleation, aggregation, phase transition, anisotropic growth, and ripening. Small green rust seeds $\left(\mathrm{Fe}(\mathrm{OHCl})_{2.55}\right)$ were generated by nucleation and crystal growth (step 1, Fig. 5b, 1 h). These seeds aggregated to form larger rod-like structures and transformed into iron hydroxide, goethite, and iron oxide hydroxide (step 2, Fig. $5 \mathrm{c}, 1.5 \mathrm{~h}$ ) and then continuously aggregated and partly transformed into oval-like hematite (step 3, Fig. 5d, 2 h). At the same time, the proceeding phase transition led to the generation and deposition of hematite. As the interfacial energy between the nanoparticles and solution was higher than that between the nanoparticles and oval-like structure, the oval-like product would continuously grow anisotropically, and cantaloupe-like hematite was formed (step 4, Fig. 5 e, $3 \mathrm{~h}$ ) [22]. With prolonged time, the cantaloupe-like hematite continuously ripened and finally formed peanutlike hematite (step 5, Fig. 5f, 6 h). From the appearance of hematite reflection peaks at $2 \mathrm{~h}$, peak intensity gradually increased, indicating the higher crystallinity of peanut-like hematite compared with other hematite types. Apart from the factors mentioned above, the attraction of crystallographic plane, hydrophobic interaction, and van der Waals forces can also affect the morphology [23]. Thus, additional studies are still needed to reveal the process and evolutionary mechanism of peanut-like hematite.

The different morphologies of powder can influence infrared optical properties. Thus, the different morphologies of hematite obtained by adjusting the contents of $\mathrm{Fe}^{3+}$ and 5-SSIPA offered a chance to study the changes in infrared optical properties. The strong absorption bands from 800 to $400 \mathrm{~cm}^{-1}$ accorded with the characteristic vibration peaks of hematite. The results showed that hematite morphology affected the peak frequencies and widths. This phenomenon was also mentioned by Hu et al. [24]. As shown in Fig. 6a, with increasing 5-SSIPA contents, the absorption peaks shifted to higher wave numbers. Figure $6 \mathrm{~b}$ demonstrates that the absorption peaks shifted to lower wave numbers with increasing $\mathrm{Fe}^{3+}$ contents. The results can widen the application of different hematite types as probes for detecting several molecules [25].

Figure 7 shows the adsorption kinetic performances. The amount of adsorbed $\mathrm{As}(\mathrm{V})$ over peanut-like hematite slightly increased with prolonged time, showing the good adsorption performance of hematite. The pseudo-second-order kinetic model was applied on the data of processing to quantitatively demonstrate the adsorption efficiency [26]:

$\mathrm{d} q / \mathrm{d} t=k_{2}\left(q_{\mathrm{e}}-q_{t}\right)^{2}$ 
Fig. 6 FT-IR spectra of the samples with different morphologies and synthesized with different component contents $\left(\mathbf{a ~ F e}{ }^{3+}\right.$ fixed at $0.02 \mathrm{~mol} ; \mathbf{b}$ 5-SSIPA fixed at $0.02 \mathrm{~mol}$ )
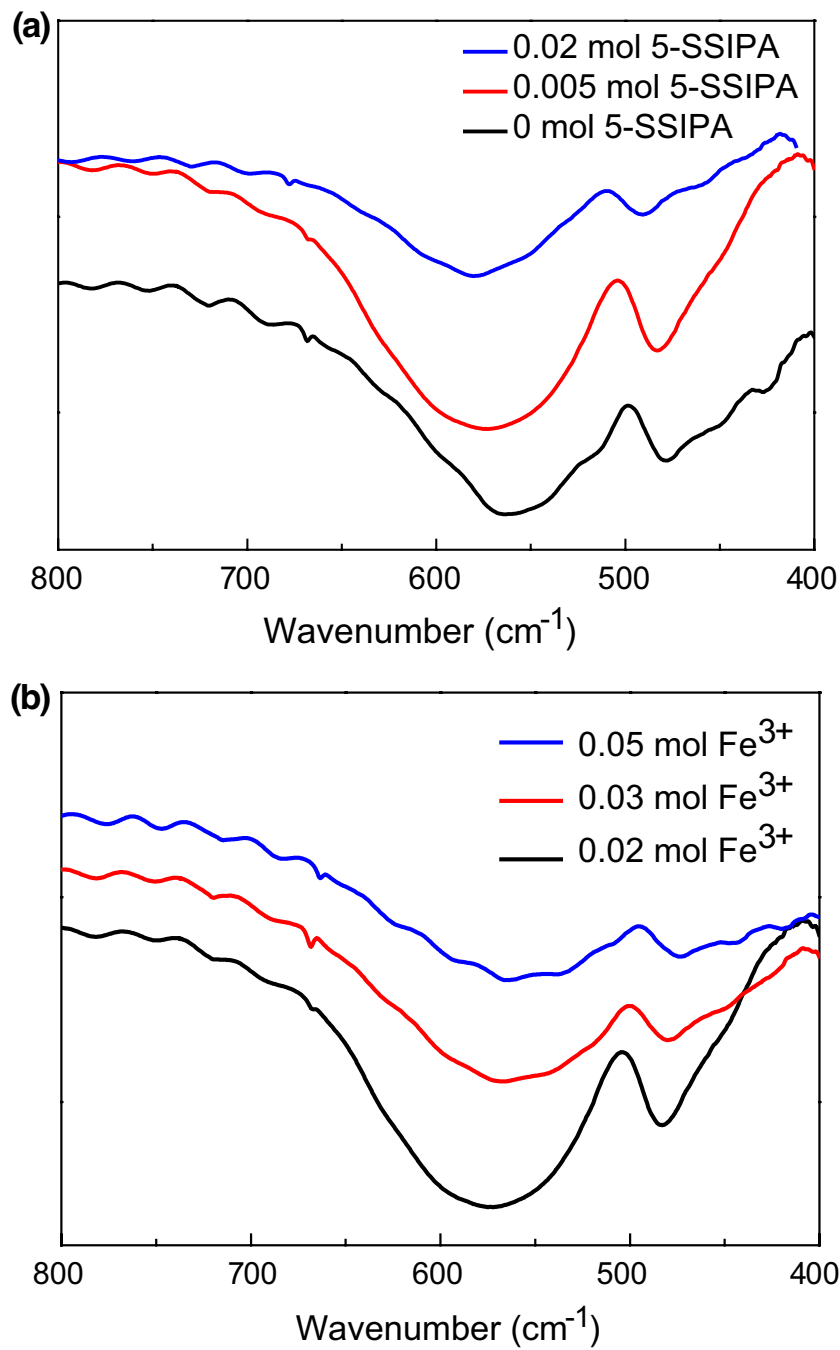

hematite nanostructures presented high performance in $\mathrm{As}(\mathrm{V})$ removal.

The selectivity of peanut-like hematite was demonstrated by competitive adsorption of other anions in the removal of $\mathrm{As}(\mathrm{V})$. Several commonly found anions in the hydrosphere, such as $\mathrm{Cl}^{-}, \mathrm{SO}_{4}{ }^{2-}, \mathrm{CO}_{3}{ }^{2-}$, and $\mathrm{PO}_{4}{ }^{3-}$, may bond to specific groups on the surface of peanut-like hematite. Hence, the selectivity of peanut-like hematite was examined by the removal of $\mathrm{As}(\mathrm{V})$ in the existence of these anions with different concentrations $(0,0.67$, and $6.7 \mathrm{mmol} / \mathrm{L}$ ). The results in Fig. 8 demonstrate that regardless of the concentrations of $\mathrm{Cl}^{-}$and $\mathrm{SO}_{4}{ }^{2-}$, the removal efficiency of $\mathrm{As}(\mathrm{V})$ was hardly affected. The existence of $\mathrm{PO}_{4}{ }^{3-}$ significantly affected the removal efficiency of $\mathrm{As}(\mathrm{V})$ over peanut-like hematite, which is possibly because of the similar structures of $\mathrm{PO}_{4}{ }^{3-}$ and $\mathrm{As}(\mathrm{V})$. The phenomena correspond to what were reported in the previous study [30]. Hence, peanut-like hematite will remove $\mathrm{PO}_{4}{ }^{3-}$ and $\mathrm{As}(\mathrm{V})$ by bonding with specific adsorption sites [31]. 

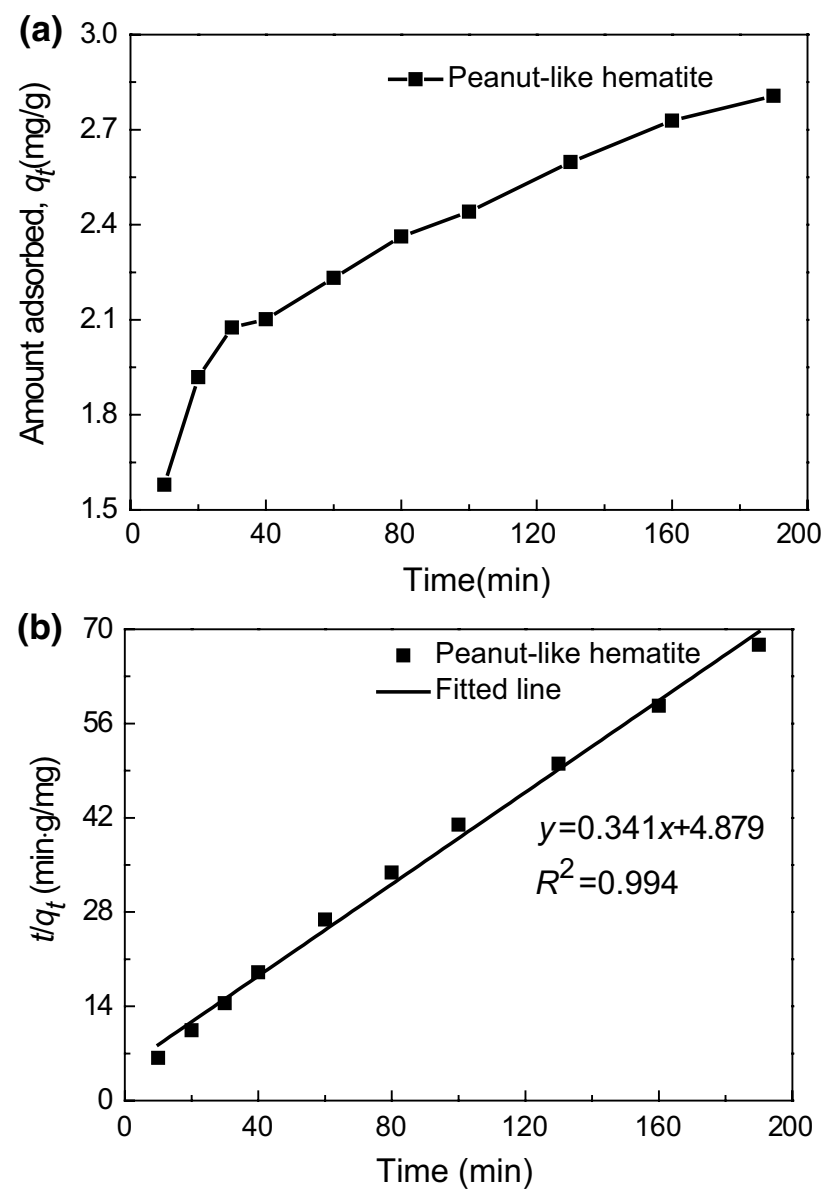

Fig. 7 a Adsorbed amount and $\mathbf{b}$ plots of pseudo-second-order kinetics of $\mathrm{As}(\mathrm{V})$ adsorption over peanut-like hematite

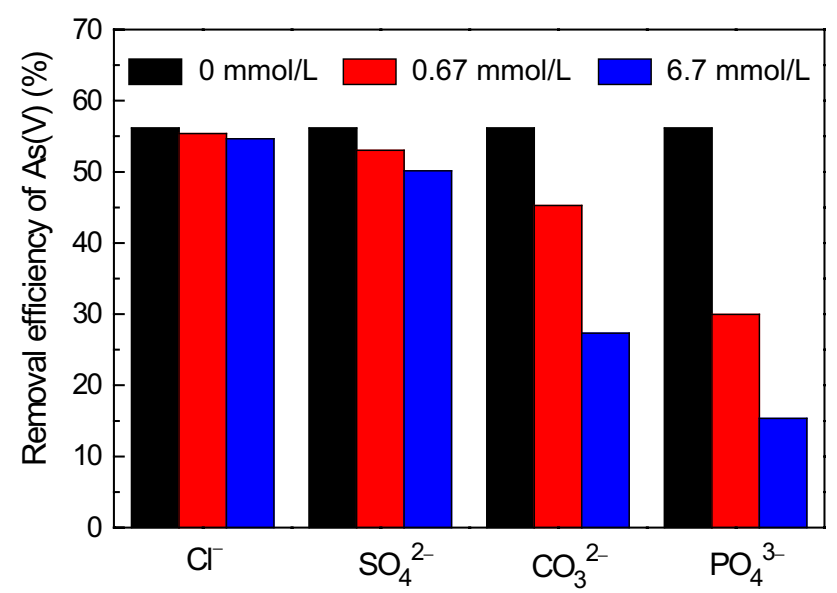

Fig. 8 Selective removal of $\mathrm{As}(\mathrm{V})$ by peanut-like hematite through the effects of anions at different concentrations

\section{Conclusions}

In summary, a new one-pot hydrothermal approach to obtain peanut-like hematite with a large BET surface area was developed in this study. The results showed that peanut-like hematite was formed by a five-step route consisting of nucleation, aggregation, phase transition, anisotropic growth, and ripening. Hematite with different morphologies can be obtained by properly controlling the hydrothermal conditions. The peanut-like hematite showed good preference and performance for $\mathrm{As}(\mathrm{V})$ removal, and the process was in accordance with the pseudo-second-order kinetic model. This study does not only serve as a reference for the synthesis of other hierarchical metal oxides or hydroxides, but also provide an opportunity to study the catalytic and electromagnetic performances of materials with different morphologies.

Acknowledgements This study was supported by the National Natural Science Foundation of China (No. 41373114); and the Program of Introducing Talents of Discipline to Universities (No. B06006).

Open Access This article is distributed under the terms of the Creative Commons Attribution 4.0 International License (http://creativeco mmons.org/licenses/by/4.0/), which permits unrestricted use, distribution, and reproduction in any medium, provided you give appropriate credit to the original author(s) and the source, provide a link to the Creative Commons license, and indicate if changes were made.

\section{References}

1. Xie X, Wang Y, Pi K et al (2015) In situ treatment of arsenic contaminated groundwater by aquifer iron coating: experimental study. Sci Total Environ 527-528:38-46

2. Pontoni L, Fabbricino M (2012) Use of chitosan and chitosanderivatives to remove arsenic from aqueous solutions-a mini review. Carbohyd Res 356:86-92

3. Kay A, Cesar I, Graetzel M (2006) New benchmark for water photooxidation by nanostructured $\alpha-\mathrm{Fe}_{2} \mathrm{O}_{3}$ films. J Am Chem Soc 128(49):15714-15721

4. Dixit S, Hering JG (2003) Comparison of arsenic(V) and arsenic(III) sorption onto iron oxide minerals: implications for arsenic mobility. Environ Sci Technol 37(18):4182-4189

5. Chang Q, Lin W, Ying WC (2010) Preparation of iron-impregnated granular activated carbon for arsenic removal from drinking water. J Hazard Mater 184(1-3):515-522

6. Liang X, Wang X, Zhuang J et al (2006) Synthesis of nearly monodisperse iron oxide and oxyhydroxide nanocrystals. Adv Funct Mater 16(14):1805-1813

7. Wu C, Yin P, Zhu X et al (2006) Synthesis of hematite ( $\alpha$-Fe2O3) nanorods: diameter-size and shape effects on their applications in magnetism, lithium ion battery, and gas sensors. J Phys Chem B 110(36):17806-17812 
8. Suber L, Imperatori P, Ausanio G et al (2005) Synthesis, morphology, and magnetic characterization of iron oxide nanowires and nanotubes. J Phys Chem B 109(15):7103-7109

9. Vayssieres L, Sathe C, Butorin SM et al (2005) One-dimensional quantum-confinement effect in $\alpha-\mathrm{Fe}_{2} \mathrm{O}_{3}$ ultrafine nanorod arrays. Adv Mater 17(19):2320-2323

10. Chen J, Xu L, Li W et al (2005) $\alpha-\mathrm{Fe}_{2} \mathrm{O}_{3}$ nanotubes in gas sensor and lithium-ion battery applications. Adv Mater 17(5):582-586

11. Zhao YM, Li YH, Ma RZ et al (2006) Growth and characterization of iron oxide nanorods/nanobelts prepared by a simple iron-water reaction. Small 2(3):422-427

12. Chen D, Gao L (2004) A facile route for high-throughput formation of single-crystal $\alpha-\mathrm{Fe}_{2} \mathrm{O}_{3}$ nanodisks in aqueous solutions of Tween 80 and triblock copolymer. Chem Phys Lett 395(4-6):316-320

13. $\mathrm{Hu} \mathrm{X}$, Yu JC, Gong J et al (2007) $\alpha-\mathrm{Fe}_{2} \mathrm{O}_{3}$ nanorings prepared by a microwave-assisted hydrothermal process and their sensing properties. Adv Mater 19(17):2324-2329

14. Atabaev TS (2015) Facile hydrothermal synthesis of flower-like hematite microstructure with high photocatalytic properties. J Adv Ceram 4(1):61-64

15. Sugimoto T, Sakata K, Muramatsu A (1993) Formation mechanism of monodisperse pseudocubic $\alpha-\mathrm{Fe}_{2} \mathrm{O}_{3}$ particles from condensed ferric hydroxide gel. J Colloid Interface Sci 159(2):372-382

16. Jia CJ, Sun LD, Luo F et al (2008) Large-scale synthesis of single-crystalline iron oxide magnetic nanorings. J Am Chem Soc 130(50):16968-16977

17. Zhong LS, Hu JS, Liang HP et al (2006) Self-assembled 3D flowerlike iron oxide nanostructures and their application in water treatment. Adv Mater 18(18):2426-2431

18. Cao CY, Qu J, Yan WS et al (2012) Low-cost synthesis of flowerlike $\alpha-\mathrm{Fe}_{2} \mathrm{O}_{3}$ nanostructures for heavy metal ion removal: adsorption property and mechanism. Langmuir 28(9):4573-4579

19. Liu ZM, Wu SH, Jia SY et al (2014) Novel hematite nanorods and magnetite nanoparticles prepared from MIL-100 $(\mathrm{Fe})$ template for the removal of As(V). Mater Lett 132:8-10

20. McIntyre NS, Zetaruk DG (1977) X-ray photoelectron spectroscopic studies of iron oxides. Anal Chem 49(11):1521-1529
21. Du Y, Jing Y, Qi M et al (2012) Fabrication and excellent conductive performance of antimony-doped tin oxide-coated diatomite with porous structure. Mater Chem Phys 133(2-3):907-912

22. Vayssieres L, Beermann N, Lindquist SE et al (2001) Controlled aqueous chemical growth of oriented three-dimensional crystalline nanorod arrays: application to iron (III) oxides. Chem Mater 13(2):233-235

23. Politi Y, Arad T, Klein E et al (2004) Sea urchin spine calcite forms via a transient amorphous calcium carbonate phase. Science 306(5699):1161-1164

24. $\mathrm{Hu} \mathrm{X}, \mathrm{Yu}$ JC (2008) Continuous aspect-ratio tuning and fine shape control of monodisperse $\alpha-\mathrm{Fe}_{2} \mathrm{O}_{3}$ nanocrystals by a programmed microwave-hydrothermal method. Adv Funct Mater 18(6):880-887

25. Eggleston CM, Khare N, Lovelace DM (2006) Cytochrome $c$ interaction with hematite $\left(\alpha-\mathrm{Fe}_{2} \mathrm{O}_{3}\right)$ surfaces. J Electron Spectrosc Relat Phenom 150(2-3):220-227

26. Hameed BH, Rahman AA (2008) Removal of phenol from aqueous solutions by adsorption onto activated carbon prepared from biomass material. J Hazard Mater 160(2-3):576-581

27. Catalano JG, Park C, Fenter P et al (2008) Simultaneous innerand outer-sphere arsenate adsorption on corundum and hematite. Geochim Cosmochim Acta 72(8):1986-2004

28. Liu Z, Chen J, Wu Y et al (2018) Synthesis of magnetic orderly mesoporous a-Fe2O3 nanocluster derived from MIL-100(Fe) for rapid and efficient arsenic(III, V) removal. J Hazard Mater 343:304-314

29. Qin FX, Jia SY, Liu Y et al (2013) Metal-organic framework as a template for synthesis of magnetic $\mathrm{CoFe}_{2} \mathrm{O}_{4}$ nanocomposites for phenol degradation. Mater Lett 101:93-95

30. Fufa F, Alemayehu E, Lennartz B (2014) Sorptive removal of arsenate using termite mound. J Environ Manag 132:188-196

31. Jain A, Loeppert RH (2000) Effect of competing anions on the adsorption of arsenate and arsenite by ferrihydrite. J Environ Qual 29(5):1422-1430 